\title{
MicroRNA-205 increases the sensitivity of docetaxel in breast cancer
}

\author{
YANG CAI, XIANG YAN, GUOQING ZHANG, WEIHONG ZHAO and SHUNCHANG JIAO \\ Department of Medical Oncology, Chinese People's Liberation Army General Hospital, Beijing 100853, P.R. China
}

Received August 27, 2014; Accepted May 20, 2015

DOI: $10.3892 / \mathrm{ol} .2015 .4030$

\begin{abstract}
Chemotherapy has been widely used in breast cancer therapy, but the efficacy of chemotherapy is intimately associated with the sensitivity of therapeutic drugs to breast cancer. Docetaxel is a first-line chemotherapeutic drug in breast cancer treatment, but further improvement to its efficacy has thus far proved difficult. microRNAs (miRs) are a class of endogenous, small, non-coding RNAs, which regulate gene expression at the post-transcriptional level. miR-205, a regulator of HER-3, is reported to be a tumor suppressor in breast cancer. In the present study, the reintroduction of miR-205 is shown to inhibit cell proliferation and clonogenic potential, and increase the sensitivity of MCF-7 and MDA-MB-231 cells to docetaxel. miR-205 also shows a synergistic effect with docetaxel in vivo. The present study provides a novel strategy to increase the sensitivity to docetaxel in breast cancer patients.
\end{abstract}

\section{Introduction}

Breast cancer is the most prevalent cancer in women around the world today (1). In 2008, it caused the most cancer-associated mortalities among women (13.7\%) (2). From 2005-2009, the age-adjusted incidence rate for breast cancer was 124.3 cases per 100,000 women per year (3). For 2012, it was estimated that 226,870 women would be diagnosed and 39,510 women would succumb to breast cancer (4). From 2002-2008, the 5 -year relative survival rate of breast cancer patients with distant cancer metastasis was very low (23.8\%) (3).

Chemotherapy is widely used in the neoadjuvant and adjuvant treatment for breast cancer, and also for advanced breast cancer. Docetaxel is a standard chemotherapy and is one of the most active drugs used in breast cancer treatment. However, it is difficult to further improve the efficacy of the drug.

Correspondence to: Professor Shunchang Jiao, Department of Medical Oncology, Chinese People's Liberation Army General Hospital, 28 Fuxing Road, Beijing 100853, P.R. China

E-mail: jiaosc@vip.sina.com

Key words: miR-205, docetaxel, breast cancer
microRNAs (miRNAs/miRs) are endogenously processed non-coding RNAs that are able to regulate the expression of genes by blockage of the translation of mRNA or by decreasing its stability. miRNA can be incorporated into RNA-induced silencing complex and guides the complex to target mRNAs, leading to post-transcription repression (5). A number of studies have found that miRNAs exert diverse functions in a broad range of biological events, which affect the sensitivity of different ex vivo cancer cell lines and nude mice models to chemotherapeutic drugs by regulating different target genes that play important roles in proliferation, cell cycle regulation, apoptosis, differentiation and angiogenesis in breast cancers (6-13). In vivo and in vitro trials have shown that miR-21, miR-10b and miR-27 can stimulate the growth of breast cancer, while miR-125a, miR-125b and miR-205 can inhibit the proliferation of breast cancer by decreasing the expression of HER-2 or HER-3. miR-206 may be associated with estrogen receptor- $\alpha$ (6-13), but the exact mechanisms remain unclear. All the aforementioned results suggest that miRNAs may act as novel potential diagnostic and treatment targets. mir-205, which directly targets the HER-3 receptor, has been found to be downregulated in breast cancer tumors (7). Recent studies have also reported that the reduced expression of miR-205 may cause docetaxel resistance in prostate cancer (14). Therefore, the present study analyzed whether docetaxel sensitivity could be increased in breast cancer therapy by reintroducing miR-205.

\section{Materials and methods}

Lentiviral constructs and transduction. To generate the miR-205 expression vector, a fragment carrying pre-miR-205 was amplified as referenced (15). Briefly, a 600 bp fragment carrying pre-miR-205 was amplified from MCF-10A genomic DNA by the Phusion ${ }^{\circledR}$ High-Fidelity DNA Polymerase enzyme (New England Biolabs, Ipswich, MA, USA) using the following PCR primers: miR-205-5.1, 5'-GAATTCCTTATCTGGGTG GCTGTTTTG-3' and miR-205-3.1, 5'-GGTACCGCGGTG CTTTTTCCAATCTGC-3'. The amplified fragment was first cloned into a pBS-hU6 vector with fusion green fluorescent protein (GFP) expression. To construct the miR-205 lentiviral expression vector, the pre-miR-205 fragment was subcloned into an FG12 vector, and then co-transfected into 293T cells with pMDLg/RRE, pRSV/rev and pHCMV-G. All the plasmids were kindly provided by $\mathrm{Mr}$. Yu (The Shanghai Cancer 
A

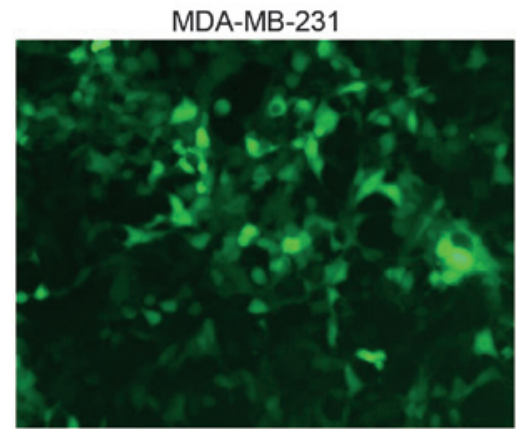

C

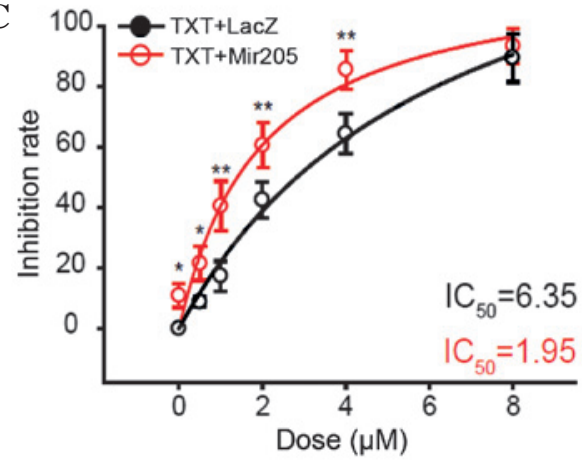

B

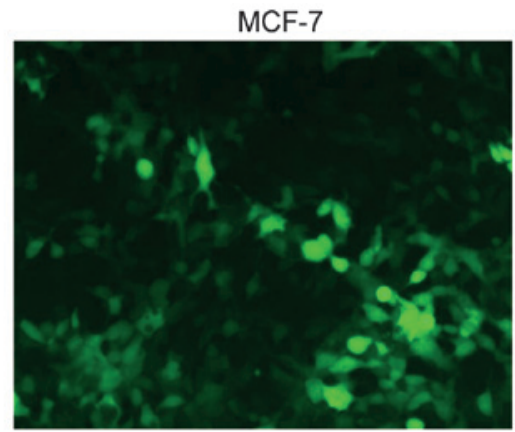

D 100

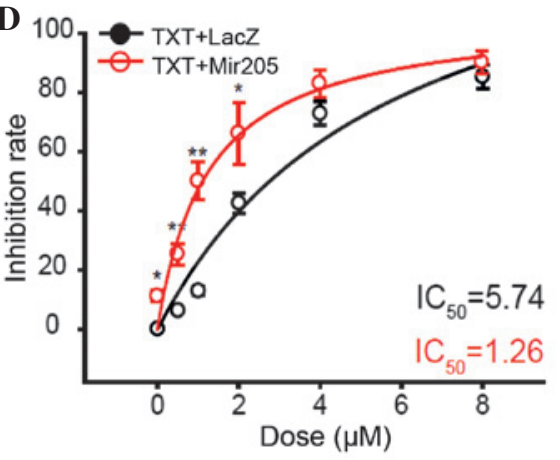

Figure 1. miR-205 overexpression in MDA-MB-231 and MCF-7 cells increases the sensitivity to docetaxel. (A and B) miR-205 expression in (A) MDA-MB-231 and (B) MCF-7 cells, as detected by green fluorescent protein expression. (C and D) miR-205 increases the sensitivity to docetaxel in (C) MDA-MB-231 and (D) MCF-7 cells. Data are expressed as the mean \pm standard deviation of six independent experiments. "P<0.05 and ${ }^{* *} \mathrm{P}<0.01$. TXT, docetaxel; IC ${ }_{50}$, half maximal inhibitory concentration; miR, microRNA.

Institute, Shanghai, China). Cell supernatants were collected at $48 \mathrm{~h}$ post-transfection and passed through a $0.22-\mathrm{mm}$ filter. The titer of purified virus was $3.0 \times 10^{8} \mathrm{IU} / \mathrm{ml}$.

Cell culture and transfection. The human breast cancer MDA-MB-231 and MCF-7 cell lines and the normal human embryonic kidney $293 \mathrm{~T}$ cell line were all obtained from the American Type Culture Collection (Manassas, VA, USA). All the cells were maintained in Dulbecco's modified Eagle's medium (DMEM)/F12 medium (Invitrogen Life Technologies, Carlsbad, CA, USA) supplemented with $10 \%$ fetal bovine serum and $1 \%$ penicillin and streptomycin (Invitrogen Life Technologies) and under a standard gas atmosphere of humidified air $/ 5 \% \mathrm{CO}_{2}$. Transient transfection was performed with lipofectamine 2000 (Invitrogen Life Technologies).

Cell proliferation assay. A CellTiter-Glo ${ }^{\circledR}$ Luminescent Cell Viability Assay kit (Promega Corporation, Madison, WI, USA) was used for cell growth measurements. A total of $1 \times 10^{4}$ cells were seeded in a 96-well plate, in $100 \mu \mathrm{l}$ medium for each well. Docetaxel (Sigma-Aldrich, St. Louis, MO, USA) was added at $0,0.5,1.0,2.0,4.0$ and $8.0 \mu \mathrm{M}$ after a $24-\mathrm{h}$ regular incubation. The cells were cultured for $36 \mathrm{~h}$, and then $100 \mu \mathrm{l} /$ well CellTiter-Glo reagent was added to measure cell growth according to the manufacturer's instructions.

Colony formation assay. The cells (1x10 $/$ well) were treated with docetaxel (Sigma-Aldrich) at a concentration of 0, 0.5, 1.0, 2.0, 4.0 and $8.0 \mathrm{mM}$ for $48 \mathrm{~h}$. Next, the cells were re-seeded at 100 cells/well in a 24 -well plate and regularly cultured in DMEM/F12 medium supplemented with $10 \%$ fetal bovine serum, and $1 \%$ penicillin and streptomycin (Invitrogen Life
Technologies) 14 days. The colonies were stained with crystal violet (Sangon Biotech Co., Ltd., Shanghai, China) and counted under a microscope (SZ61-ILST; Olympus, Tokyo, Japan). Cells were tested in four groups: The LacZ control, miR205 alone, docetaxel $(0.5 \mu \mathrm{M})$ and LacZ, and docetaxel $(0.5 \mu \mathrm{M})$ and $\mathrm{miR} 205$.

In vivo xenograft study and immunohistochemistry. The in vivo animal procedure was approved by the Animal Ethics Committee at the Chinese People's Liberation Army General Hospital (Beijing, China). The MDA-MB-231 cells and the cells stably expressing miRNA-205 were grown to $\log$ phase. A total of $1 \times 10^{7}$ microplasma free cells in $0.2 \mathrm{ml}$ phosphate-buffered saline were subcutaneously injected into the flank region of female athymic nude mice (four groups, 4 mice per group). Mice were housed and submitted to an inverse 12-h day-night cycle, with lights on at 8:30 PM, and maintained in a temperature $\left(22 \pm 1^{\circ} \mathrm{C}\right)$ and humidity $(55 \pm 5 \%)$ controlled room. Animals were housed in four different cages (Beijing ZS Dichuang Co., Ltd., Beijing, China), with 4 mice in each. The cages were filled with sterilized wood shavings, bedding and a cardboard tube for environmental enrichment. All mice were allowed free access to water and a maintenance diet (SLACOM for mouse and rat; SLAC Laboratory Animal Co., Ltd., Shanghai, China) Docetaxel (5 $\mu \mathrm{M})$ was injected directly into the xenografts from day 9 once every three days. Tumor growth was measured using calipers and body weight was monitored simultaneously. Next, formalin-fixed tissue sections were prepared for GFP detection.

RNA isolation and reverse transcription-polymerase chain reaction $(R T-P C R)$. Total RNA was extracted using TRIzol 
A

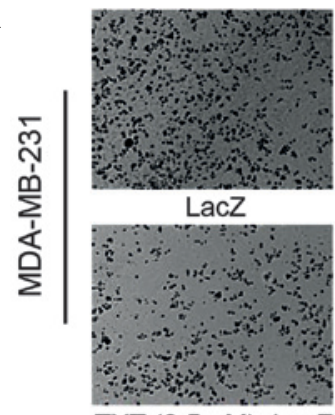

TXT $(0.5 \mu \mathrm{M})+\mathrm{LacZ}$

C

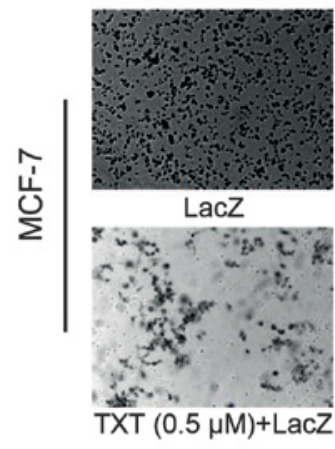

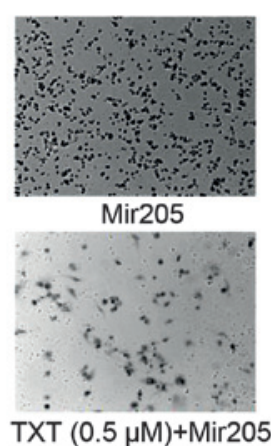
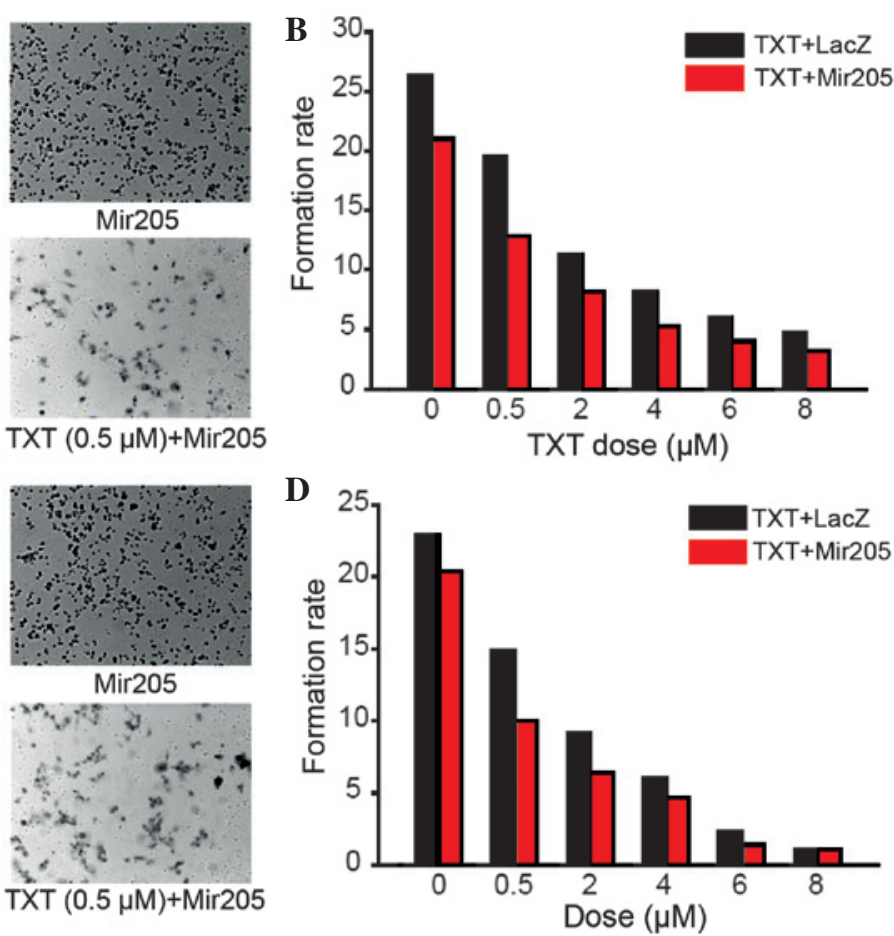

Figure 2. miR-205 inhibits colony formation in MBA-MD-231 and MCF-7 cells. (A) Colony formation in MBA-MD-231 cells treated with LacZ control, miR-205, LacZ+docetaxel and miR-205+docetaxel. (B) The colony formation rate was calculated. (C) Colony formation in MCF-7 cells treated with LacZ control, miR-205, LacZ+docetaxel and miR-205+docetaxel. (D) The colony formation rate was calculated. TXT, docetaxel; miR, microRNA.

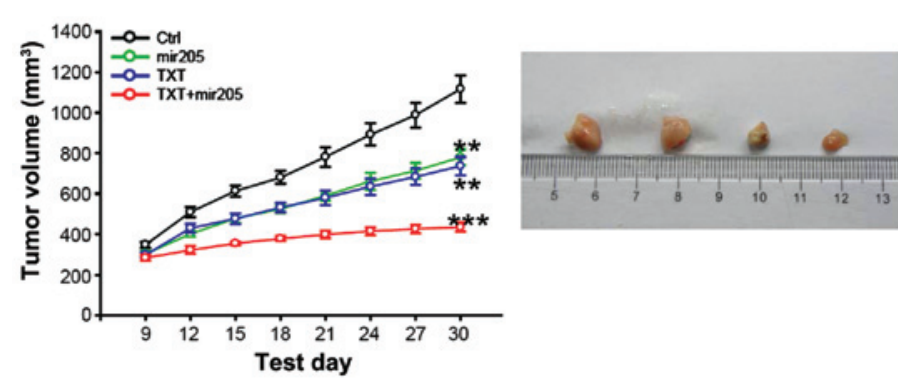

D

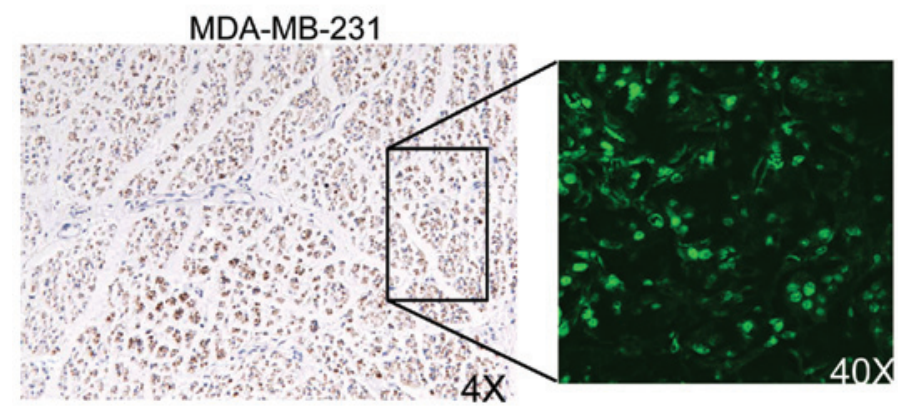

C

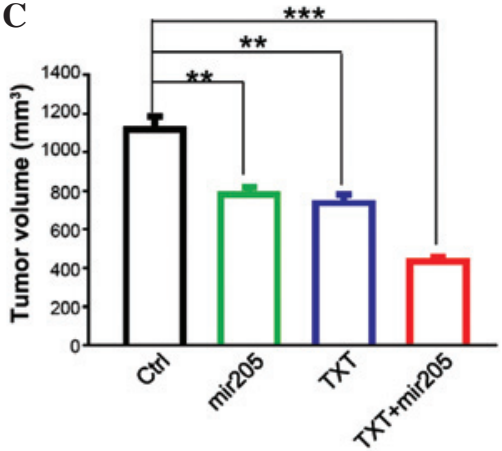

$\mathbf{E}$

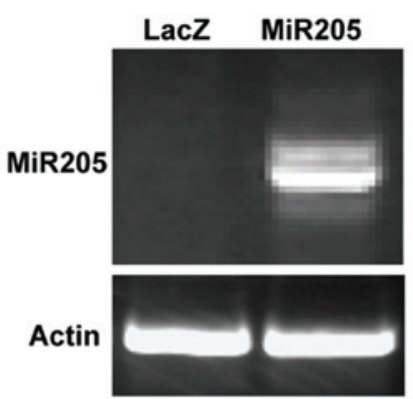

Figure 3. miR-205 inhibits tumor growth in vivo. (A) MDA-MB-231 cells were stably infected with lentiviral miR-205. Docetaxel was injected from day 9 onwards. Tumor growth was measured using calipers. Data are expressed as the mean \pm standard deviation of four independent experiments. ${ }^{* *} \mathrm{P}<0.01$ and ${ }_{* * * *} \mathrm{P}<0.05$ vs. control. (B) Tumors were resected for the control, miR-205, docetaxel, docetaxel+miR-205 treatment groups. (C) Tumor volume was measured by calipers. Data are expressed as the mean \pm standard deviation of four independent experiments. ${ }^{* *} \mathrm{P}<0.01$ and ${ }^{* * *} \mathrm{P}<0.005$ vs. control. (D) Xenograft paraffin blocks were prepared and miR-205 overexpression was confirmed by green fluorescent protein signal detection. (E) miR-205 or LacZ-infected tumors were resected for RNA extraction, and the overexpression of miR-205 was confirmed by polymerase chain reaction. TXT, docetaxel; miR, microRNA.

reagent (Invitrogen Life Technologies). RNA quality was confirmed by Nanodrop 2000 (Thermo Fisher Scientific, Inc., Waltham, MA, USA). The following PCR primers were used: Forward, 5'-TCCTCAGACAATCCATGTGC-3' and reverse, 5'-TGCCTCCTGAACTTCACTCC-3'. The miR-205 expression was detected by Platinum PCR Super 
Mix (Invitrogen Life Technologies) and amplified by Bio-Rad PCR T100 (Bio-Rad Laboratories, Inc., Hercules, CA, USA).

Statistical analysis. Results were analyzed by performing Student t-tests using Microsoft Excel 2010 (Microsoft, Redmond, WA, USA) and $\mathrm{P}<0.05$ indicated a statistically significant difference.

\section{Results}

Overexpression of miRNA-205 increases docetaxel sensitivity in breast cancer cell lines. To investigate the function of miRNA-205 in breast cancer cell lines, docetaxel sensitivity was detected in two breast cancer cell lines with or without miRNA-205 overexpression. miRNA-205 was expressed fused with GFP in the MDA-MB-231 (Fig. 1A) and MCF-7 (Fig. 1B) cell lines. The overexpression of miR-205 inhibited the cell growth of the breast cancer MDA-MB-231 (Fig. 1C) and MCF-7 (Fig. 1D) cell line. miR-205 was shown to increase cell sensitivity of MDA-MB-231 cells to $0,0.5,1,2$ and $4 \mu \mathrm{M}$ docetaxel $(\mathrm{P}=0.0453, \mathrm{P}=0.0386, \mathrm{P}=0.00526, \mathrm{P}=0.00613$ and $\mathrm{P}=0.00571$, respectively). In MCF-7 cells, cell sensitivity to docetaxel was also increased following treatment with $0,0.5$, 1 and $2 \mu \mathrm{M}$ docetaxel $(\mathrm{P}=0.0367, \mathrm{P}=0.00836, \mathrm{P}=0.00578$ and $\mathrm{P}=0.0127$, respectively).

A colony formation assay was performed to assess whether miR-205 could inhibit the clonogenic survival of the MDA-MB-231 cancer cells. Compared with the LacZ control, docetaxel alone and miR-205 alone groups, the cells treated with miR-205 combined with docetaxel showed a significantly decreased colony formation ability (Fig. 2A and B).

Similar results were acquired with another breast cancer cell line, MCF-7 (Fig. 2C and D). These results suggested that miR-205 suppresses breast cancer cell proliferation and has a synergistic effect with docetaxel treatment.

miRNA-205 has a synergistic inhibition effect with docetaxel treatment in vivo. Next, the study investigated whether miR-205 can inhibit cell growth and whether it has a synergistic effect with docetaxel in vivo. Since it is difficult to form xenograft tumors in nude mice with MCF-7 cells, the study was focused on MDA-MB-231 cells. It was found that miR-205 overexpression or docetaxel treatment inhibited tumor cell growth in vivo, and that the sensitivity to docetaxel was significantly increased when combined with miR-205 reintroduction (Fig. 3A-C). When compared with the control group, miR-205 and docetaxel treatment alone inhibited tumor growth (miR-205, P=0.00845; TXT, P=0.00648). Docetaxel exhibited a greater inhibitory effect on tumor growth when combined with miR-205 ( $\mathrm{P}=0.000641)$. miR-205 expression was confirmed by GFP detection and RT-PCR (Fig. 3D and E).

\section{Discussion}

With the occurrence of taxanes, the chemotherapeutic efficacy in breast cancer has been significantly improved. However, drug resistance and further improvements in efficacy remain great challenges in breast cancer medical oncology. Biomarker diagnosis and biotarget therapy have provided a novel direction of study since the clinical use of Herceptin, a HER-2 monoclonal antibody, in 1997. Herceptin was confirmed to provide significantly improved clinical benefits and formed the foundation of modern biotarget therapy in breast cancer. Clinical trials have confirmed that Herceptin can reduce the risk of recurrence in HER-2-positive breast cancer post-operative patients by $\sim 50 \%$, and improve the progression-free and overall survival of HER-2-positive advanced breast cancer patients $(16,17)$. Lapatinib, a multi-inhibitor, which can inhibit the tyrosin kinases of EGFR1 and EGFR2 (HER-2) was permitted to be used in Herceptin failure HER-2-positive advanced breast cancer patients by the FDA in 2001 (18-20). Inspired by the aforementioned results, research into multi-target therapy attracts much attention. Although a number of targets are currently being processed, progress and studies have thus far been primarily focused on the EGFR family.

Research efforts in human breast cancer have been focused on studying the role of altered expression. miRNA expression signatures appear to represent promise with regard to tumor characterization, and could be potential diagnostic and treatment tools. Additionally, approaches that interfere with miRNA function are also being considered. It is known that certain miRNAs confer drug resistance or sensitivity to cancer cells. However, this drug resistance is a hindrance to the effective curative treatment of solid tumors, and occurs frequently through a number of actions, including suppressed apoptosis, improved proliferation and crosstalk between different signal transduction pathways. Several miRNAs have been reported to be involved in these processes or signal pathways. In the present study, miR-205, which decreases the expression of HER-3, is suggested to function as a tumor suppressor in breast cancer development. miR-205 can increase the sensitivity of breast cancer to chemotherapeutic or biochemical drugs (5). Recently, Iorio et al identified that miR-205 can inhibit the proliferation of breast cancer cells, possibly by inhibiting the formation of heterodimer with HER-2, and increase the sensitivity of breast cancer to gefitinib and lapatinib (7). Kastl et al confirmed that miR-34a can improve the sensitivity of breast cancer to docetaxel by inhibiting the expression of the BCL-2 target gene, which is an anti-apoptotic family member (21).

The present study observed that miR-205 could improve the inhibition ability of docetaxel to the breast cancer MDA-MB-231 cell line and the MDA-MB-231 nude mouse model. The synergistic action may be due to the role of docetaxel in inhibiting the deregulation of spindle fibres (by stabilizing the microtubules in spindle fibres, arresting the cell at $\mathrm{M}$ phase and by inducing cell apoptosis), and the role of miR-205 in downregulating the post-transcriptional expression of HER-3. As observed in previous studies $(7,15)$, transferring miR-205 alone also can inhibit the proliferation of the breast cancer MDA-MB-231 cell line, which be may be ascribed to blocking of the PI3K/AKT pathway. At the same time, the present study data also showed that the synergistic action is most significant when the concentration of docetaxel is between 1 and $4 \mu \mathrm{mol} / 1$, while the concentration of miR-205 is constant. When the concentration of docetaxel is above a certain high level, the effect of miR-205 is no more significant; the reason behind this may lie in the fact that a high concentration of docetaxel can kill the entire breast cancer cell without the action of miR-205. 
In summary, the present results strongly suggested that miR-205 may have a synergistic action with docetaxel by the downregulation of the post-transcriptional expression of HER-3. Elevated miR-205 expression shows promise as a novel strategy for the treatment of HER-2-positive breast cancer.

\section{References}

1. Azambuja E, Durbecq V, Rosa DD, Colozza M, Larsimont D, Piccart-Gebhart M and Cardoso F: HER-2 overexpression/amplification and its interaction with taxane-based therapy in breast cancer. Ann Oncol 19: 223-232, 2008

2. World Health Organization: World Cancer Report 2008. http:// www.iarc.fr/en/publications/pdfs-online/wcr/2008/wcr_2008. pdf. Accessed February 26, 2011.

3. Howlader N, Noone AM, Krapcho M, Neyman N, Aminou R, Altekruse SF, Kosary CL, Ruhl J, Tatalovich Z, et al (eds): SEER Cancer Statistics Review, 1975-2009 (Vintage 2009 Populations). http://seer.cancer.gov/csr/1975_2009_pops09/. Accessed May 20, 2013.

4. Cancer Facts \& Figures 2012. American Cancer Society, Atlanta, p4, 2012.

5. He L and Hannon GJ: MicroRNAs: Small RNAs with a big role in gene regulation. Nat Rev Genet 5: 522-531, 2004.

6. Khoshnaw SM, Green AR, Powe DG and Ellis IO: MicroRNA involvement in the pathogenesis and management of breast cancer. J Clin Pathol 62: 422-428, 2009.

7. Iorio MV, Casalini P, Piovan C, Di Leva G, Merlo A, Triulzi T, Ménard S, Croce CM and Tagliabue E: MicroRNA-205 regulates HER3 in human breast cancer. Cancer Res 69: 2195-2200, 2009.

8. Meng F, Henson R, Lang M, Wehbe H, Maheshwari S, Mendell JT, Jiang J, Schmittgen TD and Patel T: Involvement of human micro-RNA in growth and response to chemotherapy in human cholangiocarcinoma cell lines. Gastroenterology 130: 2113-2129, 2006.

9. Zhu S, Si ML, Wu H and Mo YY: MicroRNA-21 targets the tumor suppressor gene tropomyosin 1 (TPM1). J Biol Chem 282: 14328-14336, 2007.

10. Zhu S, Wu H, Wu F, Nie D, Sheng S and Mo YY: MicroRNA-21 targets tumor suppressor genes in invasion and metastasis. Cell Res 18: 350-359, 2008.
11. Adams BD, Furneaux $\mathrm{H}$ and White BA: The micro-ribonucleic acid (miRNA) miR-206 targets the human estrogen receptor-alpha (ERalpha) and represses ERalpha messenger RNA and protein expression in breast cancer cell lines. Mol Endocrinol 21: 1132-1147, 2007.

12. Kondo N, Toyama T, Sugiura H, Fujii Y and Yamashita H: MiR-206 expression is down-regulated in estrogen receptor alpha-positive human breast cancer. Cancer Res 68: 5004-5008, 2008.

13. Tavazoie SF, Alarcón C, Oskarsson T, Padua D, Wang Q, Bos PD, Gerald WL and Massagué J: Endogenous human microRNAs that suppress breast cancer metastasis. Nature 451: 147-152, 2008

14. Wang N, Li Q, Feng NH, Cheng G, Guan ZL, Wang Y, Qin C, Yin CJ and Hua LX: MiR-205 is frequently downregulated in prostate cancer and acts as a tumor suppressor by inhibiting tumor growth. Asian J Androl 15: 735-741, 2013.

15. Wu H, Zhu S and Mo YY: Suppression of cell growth and invasion by miR-205 in breast cancer. Cell Res 19: 439-448, 2009.

16. Romond EH, Perez EA, Bryant J, Suman VJ, Geyer CE Jr, Davidson NE, Tan-Chiu E, Martino S, Paik S, Kaufman PA, et al: Trastuzumab plus adjuvant chemotherapy for operable HER2-positive breast cancer. N Engl J Med 353: 1673-1684, 2005.

17. Slamom DJ, Leyland-Jones B, Shak S, Fuchs H, Paton V, Bajamonde A, Fleming T, Eiermann W, Wolter J, Pegram M, et al: Use of chemotherapy plus a monoclonal antibody against HER-2 for metastatic breast cancer that overexpresses HER-2. J Engl J Med 344: 783-792, 2001.

18. Burris HA: Dual kinase inhibition in the treatment of breast cancer: initial experience with the EGFR/ErbB-2 inhibitor lapatinib. Oncologist 9 (Suppl 3): 10-5, 2004

19. Higa GM and Abraham J: Lapatinib in the treatment of breast cancer. Expert Rev Anticancer Ther 7: 1183-1192, 2007.

20. [no authors listed]: Breast cancer drug approved for new indication. Womens Health (Lond Engl) 6: 173, 2011.

21. Kastl L, Brown I and Schofield AC: MiRNA-34a is associated with docetaxel resistance in human breast cancer cells. Breast Cancer Res Treat 131: 445-454, 2012. 